\title{
An approach of faults estimation in Takagi-Sugeno fuzzy systems
}

\author{
Atef Khedher, Kamel Benothman \\ LARA Automatique, ENIT, BP 37, \\ le Belvédère, 1002 Tunis, \\ Email: khedher_atef@yahoo.fr \\ kamel.benothman@enim.rnu.tn
}

Didier Maquin

CRAN, UMR 7039, Nancy-Université, CNRS, 2, avenue de la Forêt de Haye,

54516 Vandœuvre-lès-Nancy,

Email: didier.maquin@ensem.inpl-nancy.fr

\author{
Mohamed Benrejeb \\ LARA Automatique, ENIT, BP 37, \\ le Belvédère, 1002 Tunis, \\ Email: mohamed.benrejeb.enit.rnu.tn
}

\begin{abstract}
In this work, the problem of fault detection and identification in systems described by Takagi-Sugeno fuzzy systems is studied. A proportional integral observer is conceived in order to reconstruct state and faults which can affect the system. In order to estimate actuator and sensor faults, a mathematical transformation is made to conceive an augmented system, in which the initial sensor fault appears as an actuator fault. Considering actuator fault as an unknown input, one can use an unknown inputs estimation method. The noise effect on the state and fault estimation is also minimized.
\end{abstract}

\section{INTRODUCTION}

Observers with unknown inputs [9] are used to estimate actuators faults which can be assumed to unknown inputs. This estimation can be made using a proportional integral observer [5], [8], [13], [12]. In often cases, process can be subjected to disturbances which have as origin the noises due to its environment, uncertainty of measurements and faults of sensors and/or actuators. These disturbances have harmful effects on the normal behaviour of the process and their estimation can be used to conceive a control strategy able to minimize their effects [16]. In the case of sensor faults, Edwards [3] proposes for linear systems to use a new state which is a filtrate version of the output, to conceive an augmented system in which the sensor fault appears as an unknown input. This formulation was used by [17] to make sensor faults estimation. Khedher and al. [7] were proposed an extension of this method to nonlinear Takagi-Sugeno Fuzzy systems.

The main contribution in this paper is to estimate simultaneously actuator and sensor fauls, affecting Takagi-Sugeno fuzzy systems. Sensor faults estimation is made using the extension of the mathematical transformation proposed in [3] to this kind of models.

The paper is organised as follows. Section 2 recalls an elementary background about the Takagi-Sugeno fuzzy model. The proposed method of faults estimation is the subject of section 3. A numerical example which shows the performance of the proposed approach is presented in the last section.

\section{ON THE TAKAGI-SUGENO FUZZY SYSTEMS}

Approaches using nonlinear Takagi-Sugeno Fuzzy systems, (sometimes named multiple model [6], [11]) are the object of many works in different contexts dealing with the presence of unknown inputs or parameter uncertainties [2], [4], [5], [7], [12], [14]. This model, known as Takagi-Sugeno multiple model, has been initially proposed, in a fuzzy modelling framework, by Takagi and Sugeno [15] and in a multiple model modelling framework by Johansen and Foss [6].

Each nonlinear dynamic system can be simply, described by a Takagi-Sugeno fuzzy model [15]. A Takagi-Sugeno fuzzy model, is the fuzzy fusion of many linear models, each of them represents the local system behaviour around an operating point. A Takagi-Sugeno model is described by fuzzy IF-THEN rules which represent local linear input/output relations of the non-linear system. It has a rule base of $M$ rules, each having $p$ antecedents, where the $i^{\text {th }}$ rule is expressed as

$$
\begin{array}{r}
R^{i}: \text { IF } \xi_{1} \text { is } F_{1}^{i} \text { and } \ldots \text { and } \xi_{p} \text { is } F_{p}^{i} \\
\text { THEN : }\left\{\begin{array}{l}
\dot{x}(t)=A_{i} x(t)+B_{i} u(t) \\
y=C_{i} x(t)
\end{array}\right.
\end{array}
$$

in which $i=1, \ldots, M, F_{j}^{i}(j=1, \ldots, p)$ are fuzzy sets and $\xi=\left[\xi_{1}, \xi_{2}, \ldots, \xi_{p}\right]$ is a known vector of premise variables [10] which may depend on the state, the input or the output. The final output of the normalized Takagi-Sugeno fuzzy model can be inferred as:

$$
\left\{\begin{aligned}
\dot{x}(t) & =\sum_{i=1}^{M} \mu_{i}(\xi(t))\left(A_{i} x(t)+B_{i} u(t)\right) \\
y(t) & =\sum_{i=1}^{M} \mu_{i}(\xi(t)) C_{i} x(t)
\end{aligned}\right.
$$

The weighting functions $\mu_{i}(\xi(t))$ are nonlinear and depend on the decision variable $\xi(t)$.

The weighting functions are normalized rule defined as :

$$
\mu_{i}(\xi(t))=\frac{T_{j=1}^{p} \omega_{i}(\xi(t))}{\sum_{j=1}^{M} T_{j=1}^{p} \omega_{j}(\xi(t))}
$$

where $\omega_{i}(\xi(t))$ is the grade of membership of the premise variable $\xi$ and $T$ denotes a t-norm. The weighting functions must satisfy the sum convex property expressed in the following equations :

$$
0 \leq \mu_{i}(\xi(t)) \leq 1, \quad \sum_{i=1}^{M} \mu_{i}(\xi(t))=1
$$


If, in the equation which defines the output, we impose that $C_{1}=C_{2}=\ldots=C_{M}=C$, the output of the mutiple model (2) is reduced to: $y(t)=C x(t)$ and the multiple model becomes:

$$
\begin{aligned}
& \dot{x}(t)=\sum_{i=1}^{M} \mu_{i}(\xi(t))\left(A_{i} x(t)+B_{i} u(t)\right) \\
& y(t)=C x(t)
\end{aligned}
$$

This model has been largely considered for analysis, modelling, control and state estimation of nonlinear systems [1].

\section{FAULTS ESTIMATION}

Consider the following nonlinear Takagi-Sugeno system affected by a sensor fault, actuator fault and a measurement noise described by :

$$
\begin{aligned}
\dot{x}(t) & =\sum_{i=1}^{M} \mu_{i}(\xi(t))\left(A_{i} x(t)+B_{i} u(t)+E_{i} f(t)\right) \\
y(t) & =C x(t)+F g(t)+D w(t)
\end{aligned}
$$

where $x(t) \in R^{n}$ represents the system state, $y(t) \in R^{m}$ is the measured output, $u(t) \in R^{r}$ is the system input, $f(t)$ and $g(t)$ represent respectively actuator and sensor faults which are assumed to be bounded and $w(t)$ is the measurement noise. $A_{i}, B_{i}$ and $C$ are known constant matrices with appropriate dimensions. $E_{i}, F$ and $D$ are respectively the actuator faults, the sensor faults and the noise distribution matrices which are assumed to be known. The scalar $M$ represents the number of local models.

Let us consider the new state $z \in R^{p}$ given by :

$$
\dot{z}(t)=\sum_{i=1}^{M} \mu_{i}(\xi(t))\left(-\bar{A}_{i} z(t)+\bar{A}_{i} C x(t)+\bar{A}_{i} F g(t)\right)
$$

where $-\bar{A}_{i}, \quad i \in 1, \ldots, M$ are stables matrices.

One introduce the augmented state $X=\left[\begin{array}{ll}x^{T} & z^{T}\end{array}\right]^{T}$, this state is given by :

$$
\begin{aligned}
\dot{X}(t) & \left.=\sum_{i=1}^{M} \mu_{i} \xi(t)\right)\left(A_{a i} X(t)+B_{a i} u(t)+W_{a i} e(t)\right) \\
Y(t) & =C_{a} X(t)+D_{a} w(t)
\end{aligned}
$$

with :

$$
\begin{gathered}
A_{a i}=\left[\begin{array}{cc}
A_{i} & 0 \\
\bar{A}_{i} C & -\bar{A}_{i}
\end{array}\right], B_{a i}=\left[\begin{array}{c}
B_{i} \\
0
\end{array}\right], D_{a}=\left[\begin{array}{c}
D \\
0
\end{array}\right] \\
C_{a}=\left[\begin{array}{cc}
C & 0 \\
0 & I
\end{array}\right], W_{a i}=\left[\begin{array}{cc}
E & 0 \\
0 & \bar{A}_{i} F
\end{array}\right], e=\left[\begin{array}{c}
f \\
g
\end{array}\right]
\end{gathered}
$$

The structure of the chosen observer is :

$$
\begin{aligned}
& \dot{\hat{X}}(t)=\sum_{i=1}^{M} \mu_{i}(\xi(t))\left(A_{a i} \hat{X}(t)+B_{a i} u(t)+W_{a i} e(t)+\right. \\
& \left.K_{i}(Y(t)-\hat{Y}(t))\right) \\
& \hat{e}(t)=\sum_{i=1}^{M} \mu_{i}(\xi(t))\left(L_{i}(Y(t)-\hat{Y}(t))\right) \\
& \hat{Y}(t)=C_{a} \hat{X}(t)
\end{aligned}
$$

where $\hat{X}(t)$ is the estimated system state, $\hat{e}(t)$ represents the estimated fault, $\hat{Y}(t)$ is the estimated output, $K_{i}$ are the local model proportional observer gains and $L_{i}$ are the local model integral gains to be computed.

Let us define the state estimation error $\tilde{x}(t)$ and the fault estimation error $\tilde{e}(t)$ :

$$
\tilde{x}(t)=X(t)-\hat{X}(t) \text { and } \tilde{e}(t)=e(t)-\hat{e}(t)
$$

The dynamics of these errors can be expessed as follow :

$\dot{\tilde{x}}(t)=\sum_{i=1}^{M} \mu_{i}(\xi(t))\left(\left(A_{a i}-K_{i} C_{a}\right) \tilde{x}(t)+W_{a i} \tilde{f}(t)+K_{i} D_{a} w(t)\right)$

$$
\dot{\tilde{e}}(t)=\dot{e}(t)-\sum_{i=1}^{M} \mu_{i}(\xi(t))\left(L_{i} C_{a} \tilde{x}(t)-L_{i} D_{a} w(t)\right)
$$

The following matrices are introduced :

$$
\varphi=\left[\begin{array}{ll}
\tilde{x}^{T} & \tilde{e}^{T}
\end{array}\right]^{T} \text { and } \varepsilon=\left[\begin{array}{ll}
w^{T} & \dot{e}^{T}
\end{array}\right]^{T}
$$

The equations (12) and (13) can be written :

$$
\dot{\varphi}=A_{m} \varphi+B_{m} \varepsilon
$$

with :

$$
A_{m}=\sum_{i=1}^{M} \mu_{i}(\xi(t)) \tilde{A}_{i} \text { and } B_{m}=\sum_{i=1}^{M} \mu_{i}(\xi(t)) \tilde{B}_{i}
$$

where :

$$
\tilde{A}_{i}=\left[\begin{array}{cc}
A_{a i}-K_{i} C_{a} & W_{a i} \\
-L_{i} C_{a} & 0
\end{array}\right], \tilde{B}_{i}=\left[\begin{array}{cc}
-K_{i} D_{a} & 0 \\
-L_{i} D_{a} & I
\end{array}\right]
$$

The matrix $I$ is the identity matrix with appropriate dimensions. In order to analyse the convergence of the generalized estimation error $\varphi(t)$, let us consider the following quadratic Lyapunov candidate function $V(t)$ :

$$
V(t)=\varphi(t)^{T} P \varphi(t)
$$

where $P$ denotes a positive definite matrix.

The problem of robust state and fault estimation is reduced to find the gains $K$ and $L$ of the observer to ensure an asymptotic convergence of $\varphi(t)$ towards zero if $\varepsilon(t)=0$ and to ensure a bounded error in the case where $\varepsilon(t) \neq 0$, i.e. :

$$
\begin{aligned}
& \lim _{t \rightarrow \infty} \varphi(t)=0 \quad \text { for } \varepsilon(t)=0 \\
& \|\varphi(t)\|_{Q_{\varphi}} \leq \lambda\|\varepsilon(t)\|_{Q_{\varepsilon}} \quad \text { for } \varepsilon(t) \neq 0 \text { and } e(0)=0
\end{aligned}
$$

where $\lambda>0$ is the attenuation level. To satisfy the constraints (19), it is sufficient to find a Lyapunov function $V(t)$ such that

$$
\dot{V}(t)+\varphi^{T} Q_{\varphi} \varphi-\lambda^{2} \varepsilon^{T} Q_{\varepsilon} \varepsilon<0
$$

where $Q_{\varphi}$ and $Q_{\varepsilon}$ are two positive definite matrices. In order to simplify the notations, the time index $(t)$ will be omitted henceforth. The inequality (20) can also be written as :

$$
\psi^{T} \Omega \psi<0
$$

with :

$$
\psi=\left[\begin{array}{l}
\varphi \\
\varepsilon
\end{array}\right], \Omega=\left[\begin{array}{cc}
A_{m}^{T} P+P A_{m}+Q_{\varphi} & P B_{m} \\
B_{m}^{T} P & -\lambda^{2} Q_{\varepsilon}
\end{array}\right]
$$


The inequality (21) holds if $\Omega<0$. The matrices $A_{m}$ and $B_{m}$ can be written as :

$$
A_{m}=\tilde{A}_{m}-\tilde{K}_{m} \tilde{C} \text { and } B_{m}=-\tilde{K}_{m} \tilde{D}+\tilde{I}
$$

with : $\tilde{A}_{m}=\sum_{i=1}^{M} \mu_{i}(\xi) \tilde{A}_{m i}$ and $\tilde{K}_{m}=\sum_{i=1}^{M} \mu_{i}(\xi) \tilde{K}_{m i}$

where : $\tilde{K}_{m i}=\left[\begin{array}{c}K_{i} \\ L_{i}\end{array}\right]$ and $\tilde{A}_{m i}=\left[\begin{array}{cc}A_{a i} & W_{a i} \\ 0 & 0\end{array}\right]$

With the following changes of variables $G_{m}=P \tilde{K}_{m}$ and $m=\lambda^{2}$, the matrix $\Omega$ can be put in the following form :

$\Omega=\left[\begin{array}{cc}P \tilde{A}_{m}+\tilde{A}_{m}^{T} P-G_{m} \tilde{C}-\tilde{C}^{T} G_{m}^{T}+Q_{\varphi} & -G_{m} \tilde{D}+P \tilde{I} \\ \tilde{I}^{T} P-\tilde{D}^{T} G_{m}^{T} & -m Q_{\varepsilon}\end{array}\right]$

As $\Omega=\sum_{i=1}^{M} \mu_{i}(\xi) \Omega_{i}$, the negativity of $\Omega$ is assured if, $\Omega_{i}<$ $0 \forall i \in\{1 \ldots M\}$ with :

$\Omega_{i}=\left[\begin{array}{cc}P \tilde{A}_{m i}+\tilde{A}_{m i}^{T} P-G_{i} \tilde{C}-\tilde{C}^{T} G_{i}^{T}+Q_{\varphi} & -G_{i} \tilde{D}+P \tilde{I} \\ \tilde{I}^{T} P-\tilde{D}^{T} G_{i}^{T} & -m Q_{\varepsilon}\end{array}\right]$

and $G_{i}=P \tilde{K}_{m i}$. Solving LMI's $\Omega_{i}<0$ leads to determine the matrices $P$ and $G_{i}$ and the scalar $m$. The gain matrices are then deduced : $\tilde{K}_{m i}=P^{-1} G_{i}$. The observer design is summarized by the following theorem:

Theorem: The system (15) describing the time evolution of the state estimation error $\tilde{x}$ and the fault estimation error $\tilde{f}$ is stable and the $\mathcal{L}_{2}$-gain of the transfer from $\varepsilon(t)$ to $\varphi(t)$ is bounded, if there exists a symmetric, positive definite matrix $P$, gain matrices $G_{i}, i \in\{1 \ldots M\}$ and a positive scalar $m$ such that the following LMI are verified:

$\Omega_{i}=\left[\begin{array}{cc}P \tilde{A}_{m i}+\tilde{A}_{m i}^{T} P-G_{i} \tilde{C}-\tilde{C}^{T} G_{i}^{T}+Q_{\varphi} & -G_{i} \tilde{D}+P \tilde{I} \\ \tilde{I}^{T} P-\tilde{D}^{T} G_{i}^{T} & -m Q_{\varepsilon}\end{array}\right]$

The observer gains (proportional and integral gains) are computed using $\tilde{K}_{m i}=P^{-1} G_{i}$ and the attenuation level is given by $\lambda=\sqrt{m}$.

\section{ILlustrative EXAMPLE}

Consider the nonlinear system described by a TakagiSugeno model with two local models, four states and four outputs, where $\xi(t)=u(t)$. Its structure is given by :

$$
\begin{aligned}
\dot{x}(t) & =\sum_{i=1}^{2} \mu_{i}(u(t))\left(A_{i} x(t)+B_{i} u(t)+E_{i} f(t)\right) \\
y(t) & =C x(t)+F g(t)+D w(t)
\end{aligned}
$$

The system matrices are defined as below :

$$
\begin{gathered}
A_{1}=\left[\begin{array}{cccc}
-0.3 & -3 & -0.5 & 0.1 \\
-0.7 & -5 & 2 & 4 \\
2 & -0.5 & -5 & -0.9 \\
-0.7 & -2 & 1 & -0.9
\end{array}\right], B_{1}=\left[\begin{array}{cc}
1 & 2 \\
5 & 1 \\
4 & -3 \\
1 & 2
\end{array}\right] \\
A_{2}=\left[\begin{array}{cccc}
-0.2 & -3 & -0.6 & 0.3 \\
-0.6 & -4 & 1 & -0.6 \\
3 & -0.9 & -7 & -0.2 \\
-0.5 & -1 & -2 & -0.8
\end{array}\right], B_{2}=\left[\begin{array}{cc}
4 & 6 \\
0 & 0 \\
-4 & 2 \\
7 & 6
\end{array}\right] \\
E_{1}=B_{1}, \quad E_{2}=B_{2}, \quad C=I
\end{gathered}
$$

$$
D=\left[\begin{array}{cc}
0.5 & 0.5 \\
0.2 & 0.2 \\
0.1 & 0.1 \\
0 & 0.1
\end{array}\right] \text { and } F=\left[\begin{array}{cc}
3.25 & 5 \\
0 & 0.5 \\
-3.25 & 1.75 \\
5.75 & 5
\end{array}\right]
$$

The chosen weighting functions depends on the two inputs of the system. They have been created on the basis of Gaussian membership functions. Figure 1 shows their time-evolution showing that the system is clearly nonlinear since $\mu_{1}$ and $\mu_{2}$ are not constant functions.

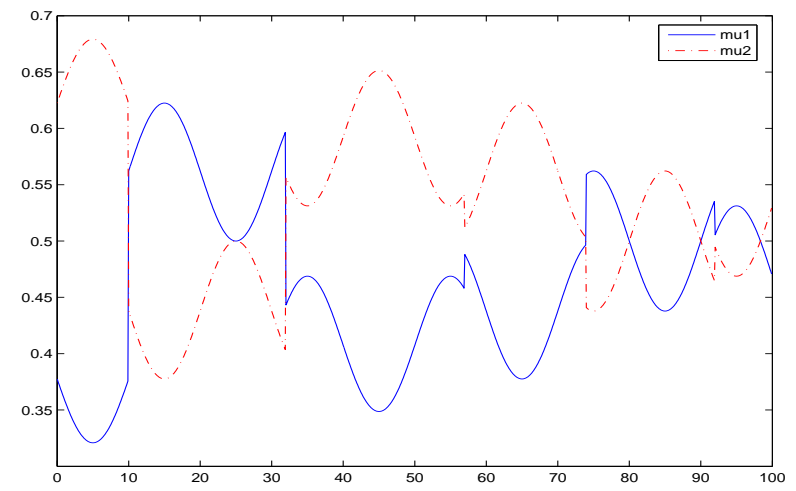

Fig. 1. Weighting functions

Considering $u(t)=\left[\begin{array}{ll}u_{1}(t) & u_{2}(t)\end{array}\right]^{T}$, the signal $u_{1}(t)$ is a telegraph type signal whose amplitude is belongs to the interval $[0,0.5]$.

The signal $u_{2}(t)$ is defined by $u_{2}(t)=0.3+0.1 \sin (\pi t)$. The actuator fault $f(t)$ is made up of two components : $f(t)=\left[\begin{array}{ll}f_{1}(t) & f_{2}(t)\end{array}\right]^{T}$ with :
$f_{1}=\left\{\begin{array}{l}0, t \leq 6 \mathrm{sec} \\ \sin (0.5 \pi t), t>6 \mathrm{sec}\end{array}, f_{2}=\left\{\begin{array}{l}0, t \leq 10 \mathrm{sec} \\ 0.4, t>10 \mathrm{sec}\end{array}\right.\right.$ and the sensor fault $g(t)$ is defined as follows : $g(t)=\left[\begin{array}{ll}g_{1}(t) & g_{2}(t)\end{array}\right]^{T}$ with : $g_{1}=\left\{\begin{array}{l}0, t \leq 20 \mathrm{sec} \\ \sin (0.3 \pi t), t>20 \mathrm{sec}\end{array}, g_{2}=\left\{\begin{array}{l}0, t \leq 35 \mathrm{sec} \\ 0.6, t>35 \mathrm{sec}\end{array}\right.\right.$

Choosing $Q_{\varphi}=Q_{\varepsilon}=I, \bar{A}_{1}=10 * I$ and $\bar{A}_{2}=15 * I$, the $\mu, K_{1}, K_{2}, L_{1}$ and $L_{2}$ can be computed by solving the LMI (26).

The simulation results are shown in the figures 2 to 4 . the proposed method provides good estimates of the system state (one present the states error of estimation of system (6) and simultaneously actuator and sensor faults. The proposed method allows to estimate faults even in the case of faults varying in the time. Figures 3 and 4 show the effectivnes of the method for faults having sinusoidal form.

\section{CONClusion}

This paper has presented an estimation method of sensor and actuator faults. A mathematical transformation is used to considerate sensor fault as an unknown input to an augmented system. This reconstruction is made for nonlinear systems represented by Takagi-Sugeno fuzzy models. This work include the analysis of the noise effect on measures, the proposed approche let possible to minimise the noise effect in the faults 

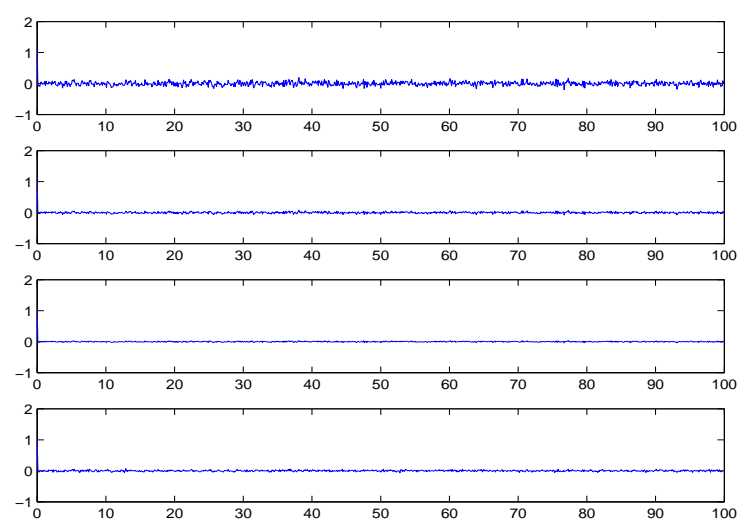

Fig. 2. State error estimation
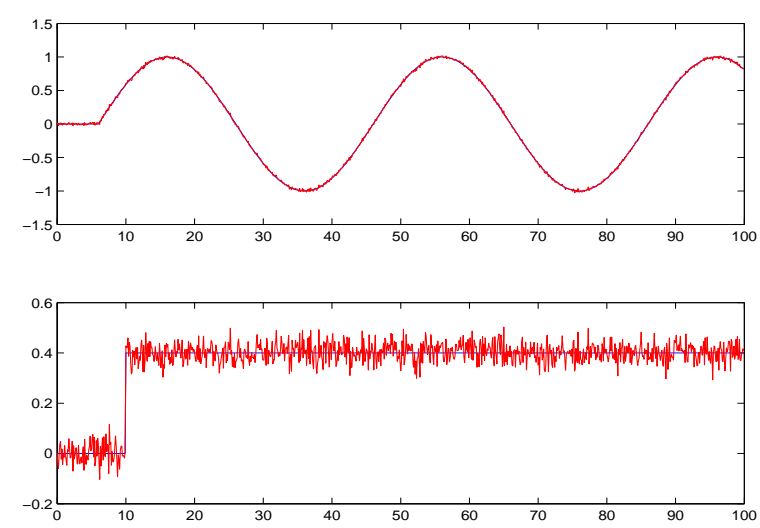

Fig. 3. Actuator faults and their estimation
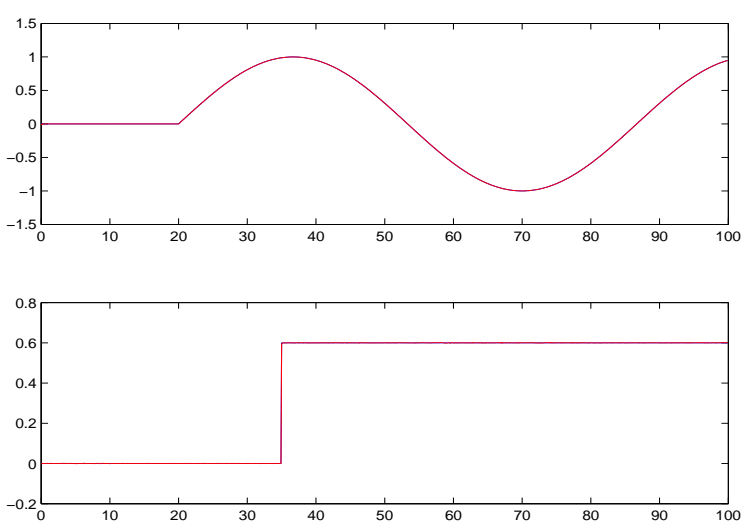

Fig. 4. Sensor faults and their estimation

estimation. The proposed method uses a proportionnal integral observer which is able to estimate simultaneously the system state and the faults. The observer design is formulated in term of linear matrices inequalities (LMI). Small size example have illustrated the efficiency of the proposed approach. It is possible to use fault estimates to conceive a fault tolerant control able to minimise its effect on the system behaviour.

\section{REFERENCES}

[1] A. Akhenak, M. Chadli, J. Ragot and D. Maquin, Design of observers for Takagi-Sugeno fuzzy models for Fault Detection and Isolation. 7th IFAC SAFEPROCESS'09, Barcelona, Spain, June 30th - July 3rd, 2009.

[2] A. Akhenak, M. Chadli, J. Ragot and D. Maquin, Design of sliding mode unknown input observer for uncertain Takagi-Sugeno model. 15th Mediterranean Conference on Control and Automation, MED'07, Athens, Greece, June 27-29, 2007.

[3] C. Edwards, A comparison of sliding mode and unknown input observers for fault reconstruction. IEEE Conference on Decision and Control, vol. 5, pp. 5279-5284, 2004.

[4] Y. Guan and M. Saif A novel approach to the design of unknown input observers. IEEE Trans on Automatic Control, AC-36, n. 5, pp. 632-635, 1991.

[5] D. Ichalal, B. Marx, J. Ragot and D. Maquin, Simultaneous state and unknown inputs estimation with PI and PMI observers for Takagi-Sugeno model with unmeasurable premise variables. 17th MED'09, Thessaloniki, Greece, June 24-26, 2009.

[6] T.A. Johansen and A.B. Foss, Nonlinear local model representation for adaptive systems. Singapore International Conference on Intelligent Control and Instrumentation, Singapore, February 17-21, 1992.

[7] A. Khedher, K. Benothman, D. Maquin and M. Benrejeb, State and sensor faults estimation via a proportional integral observer. 6th SSD'09 March 23-26, Djerba, Tunisia, 2009.

[8] A. Khedher, K. Benothman, D. Maquin and M. Benrejeb, State and unknown input estimation via a proportional integral observer with unknown inputs. 9th STA'2008 December 20-23, Sousse, Tunisia, 2008.

[9] N. Kobayashi and T. Nakamizo, An observer design for linear systems with unknown inputs, Internation Journal of Control, vol. 35, pp. 605-619, 1982.

[10] J. Korbicz, J. Koscielny, Z. Kowalczuk, and W. Cholewa, Fault diagnosis. Models, Artificial Intelligence, Applications. Springer-Verlag, Berlin, 2004.

[11] R. Murray-Smith and T. Johansen, Multiple model approaches to modeling and control. Taylor and Francis, London, 1997.

[12] R. Orjuela, B. Marx, J. Ragot and D. Maquin, On the simultaneous state and unknown inputs estimation of complex systems via a multiple model strategy. IET Control Theory \& Applications, 3(7):877-890, 2009.

[13] R. Orjuela, B. Marx, J. Ragot and D. Maquin, Proportional-Integral observer design for nonlinear uncertain systems modelled by a multiple model approach 47th IEEE Conference on Decision and Control, Cancun, Mexico, December 9-11, 2008.

[14] R. Sharma and M. Aldeen, Estimation of unknown disturbances in nonlinear systems. Control 2004, University of Bath, UK, September 6-9, 2004.

[15] T. Takagi and M. Sugeno. Fuzzy identification of systems and its application to modeling and control. IEEE Transaction Systems, Man and Cybernetics, 15(1):116132, 1985.

[16] M. Witczak, L. Dziekan, V. Puig and J. Korbicz. A fault-tolerant control strategy for Takagi-Sugeno fuzzy systems. In proceeding of the 17th World Congress The International Federation of Automatic Control Seoul, Korea, July 6-11, 2008

[17] K. Zhang, B. Jiang, V. Coquempot, Adaptive Observer-based Fast Fault Estimation. Internation Journal of Control, Automation, and systems Vol. 6 no 3 , pp 320-326 june 2008 . 